\title{
Dent Disease
}

National Cancer Institute

\section{Source}

National Cancer Institute. Dent Disease. NCI Thesaurus. Code C123260.

An X-linked, recessive disorder of the proximal renal tubules that presents during

childhood, and is characterized by low-molecular weight proteinuria, hypercalciuria,

hypophosphatemia rickets, nephrocalcinosis, nephrolithiasis, and progressive kidney

failure. 